\title{
Die Rolle des Radiointerviews im Landeskundeunterricht Ein Mediascher in Paraguay
}

\section{Ioana CUSIN}

Assist. Dr.; Universität Bukarest; E-Mail: ioana.cusin@1ls.unibuc.ro

\begin{abstract}
The tendency to apply media in regional studies for students of German as a foreign language (Deutsch als Fremdsprache, DaF) is no longer a novelty. Thus, we see media applied as such in multiple different forms, like videos, press releases, radio segments or online statements. This contribution is focused on the radio interview, as a constitutive part of the regional studies' courses for DaF students of Germanistik in Romania. As a starting point serves the assumption, that the application of visual media can be enhanced through dialogue sequences, which further highlight the subject of the course. Through chosen interviews, that have been aired by the state channels, students can become familiar with such issues, that affect the German minority in Romania. The authenticity that is being sought for, will result from the primary situation of the interviewer and the interviewee communicating, as well as through the utilization of Rumäniendeutsch as the standard language of the German minority, living in Romania. Thus can cultural, social, historical and linguistic phenomena that are specific for the local German speaking population and that are (re)constructing the cultural inheritance of Germans in Romania, be regarded in greater detail.
\end{abstract}

Keywords: Radio interview, DaF, regional studies, German minority, Rumäniendeutsch 


\section{Einführung}

Eine persönliche Beobachtung erwies, dass das Radiointerview (RI) als journalistisches Hörfunkprodukt in den DaF-Landeskundeunterricht an der Bukarester Universität noch nicht so oft eingesetzt wird. Anstelle von solchen nichtdidaktisierten Hörsequenzen tritt meistens der schriftliche, faktenzentrierte Text auf. Demzufolge fokussiert sich der vorliegende Beitrag auf das Radiointerview als konstitutiver Bestandteil des Landeskundeunterrichts für Germanistik-/DaF-Studierende in Rumänien, die ein Sprachniveau zwischen B1-B2 erreicht haben. Ausgangspunkt hierfür bildet die Annahme, dass der Einsatz von visuellen Medien auch durch derartige Dialog-Sequenzen ergänzt werden kann, die zum einen das Unterrichtsthema akustisch beleuchten, zum anderen, durch die übliche Rollenverteilung im Interview und durch den (un)bewussten Gebrauch der Varietät, dem Gespräch Authentizität verleihen und somit für die Studierenden attraktiv sind. Dies soll wiederum im Endeffekt zu einem leichteren, effizienteren Wahrnehmungsprozess ,der anderen Kultur ${ }^{1 " ~}$ beitragen.

\section{Landeskunde: interkulturell und/oder diskursiv?}

Es besteht inzwischen kein Zweifel mehr, dass der Begriff "Landeskunde" semantisch nicht abdeckend ist und dass er die Facetten des Kulturdiskurses nicht einheitlich erfassen kann. Die Debatte um eine passende Fachbenennung und Umstellung ist noch lange nicht ausgeklungen, sodass in vielen Ländern der Terminus „Landeskunde“ weiterhin das Fach über die Kultur, Geschichte, Geografie bezeichnet, so auch in Rumänien.

Die bekannten Ansätze in diesem Bereich reichen von der kognitiven, kommuntiven und interkulturellen bis hin zur

${ }^{1}$ Gemeint ist hier die rumäniendeutsche Kultur, die hierzulande durch Angehörige der deutschen Minderheit vertreten ist. 
diskursiven Landeskunde. Wenn der interkulturelle Ansatz über den faktenbezogenen und kommunikativen Ansatz hinausgeht, stellt Altmayer (2017: 10) fest, dass nun der Paradigmenwechsel im Fach Landeskunde im Fokus steht und nicht mehr die alten Ansätze ${ }^{2}$. Erwartet wird demnach vom Fach Landeskunde auf „Kulturstudien“ bzw. „kulturbezogenes Lernen“ umzusteigen.

Ausgehend vom angloamerikanischen Modell der cultural studies, sind die kulturellen Deutungsmuster und die diskursive Landeskunde weniger auf das Vermitteln von Fakten ausgerichtet, sondern wichtig ist dabei die Art und Weise, wie über diese gesprochen und verhandelt wird bzw. ihre Relevanz für den deutschsprachigen Kulturraum ${ }^{3}$. Aus diesem Blickwinkel bringen Kulturen keine Vertreter mit kulturtypischen Eigenschaften hervor. Der Wert liegt vielmehr in der Individualität des Einzelnen in Bezug auf Sprache, Handeln, Glauben, Denken, Argumetieren usw. Resümierend kann mit Senghaas (2002: 9) behauptet werden, dass „Wer immer sich heute anschickt, interkulturelle Dialoge zu inszenieren, sollte die real existierende Kulturwelt, nicht also die Fiktion von homogenen Kulturen zum Ausgangspunkt nehmen"4.

Die interkulturelle Perspektive nimmt sich daher vor, mögliche Missverständnisse und Vorurteile abzubauen, die aus der Begegnung zwischen Vertretern verschiedener Kulturkreise resultieren können. Folglich erfüllt dieser Ansatz eine Brückenfunktion zwischen dem Eigenen und dem Fremden, sodass den Lernenden die Möglichkeit geboten wird, mit ihren eigenen Weltvorstellungen und Erfahrungen in den Lernprozess

2 Altmayer, Claus: Landeskunde im Globalisierungskontext. Wozu noch Kultur im DaF-Unterricht? In: Haase, Peter/Michaela Höller (Hgg.): Kulturelles Lernen im DaF-/DaZ-Unterricht. Paradigmenwechsel in der Landeskunde. Göttingen 2017.

3 Ebd., S. 10.

${ }^{4}$ Senghaas, Dieter: Kulturelle Globalisierung - ihre Kontexte, ihre Varianten. 2002; unter http:/www.bpb.de/apuz/27019/kulturelle-globalisierung-ihre-kontexte-ihre-varianten? $\mathrm{p}=$ all; 10.05.2021. 
einzusteigen und sie für die Unterschiede zwischen den Kulturen zu sensibilisieren ${ }^{5}$.

Für den vorliegenden Beitrag wurden beide Ansätze herangezogen, da diese, wie oben beschrieben, nicht als widersprüchlich gedeutet werden müssen, sondern sich meiner Ansicht nach komplementär erweisen.

\section{Das journalistische RI von Radio Romania als authentisches Instrument der Sprach- und Kulturförderung}

Um auf den Ausgangspunkt des Beitrags zurückzukommen, möchte ich an dieser Stelle auf den authentischen Wert des journalistischen Interviews im Rahmen der deutschen Sendung ${ }^{6}$ von Radio Romania verweisen - ein Instrument, dessen Zweck weit über die Vermittlung von Informationen hinausreicht und, durch den Gebrauch der rumäniendeutschen Varietät ${ }^{7}$ und durch die interviewten Personen, zur Sprach- und Kulturförderung innerhalb der deutschen Minderheit beiträgt.

5 Rösler, Dietmar: Deutsch als Fremdsprache. Eine Einführung. Stuttgart 2012, S. 82.

${ }^{6}$ Informationen über die deutsche Minderheit in Rumänien vermittelt die deutsche Sendung des Rumänischen Rundfunks (RR) über ihre Bukarester Redaktion und deren Mitarbeiter in Temeswar/Timișoara, Hermannstadt/Sibiu und Kronstadt/Braşov. Vgl. http://www.radiobukarest.ro; 10.05.2021.

7 Ausführlicher dazu Lăzărescu, Ioan: Rumäniendeutsch - eine eigenständige, jedoch bessere Varietät der deutschen Sprache. In: SchneiderWiejowski, Karina/Birte Kellermeier-Rehbein/Jakob Haselhuber (Hgg.): Vielfalt, Variation und Stellung der deutschen Sprache. Berlin 2013, S. 369-389. Diese Varietät ist in Rumänien nicht nur Kirchen-, Literatur- oder Unterrichtssprache, sondern auch Mediensprache z.B. im öffentlich-rechtlichen Fernsehen und Rundfunk innerhalb der Sendung für die deutsche Minderheit. 
Beim Radiointerview geht es um einen gesprochensprachlichen $^{8}$ (akustischen) Text, der dem Publikum (den Zuhörern) via Radio vermittelt wird. Da das gesprochene Wort im Radio das Sagen hat, gehört das Interview als Genre zu den beliebtesten Darstellungsformen in diesem Bereich. Meistens ist es so, dass der Dialog als grundlegende Komponente der deutschen Sendung auftritt. Durch den Einsatz dialogischer Kommunikation wird im Medium die angestrebte Authentizität erreicht, was letztendlich auf den Zuhörer positiv wirkt, insofern dieser die Information direkt vom Interviewten vermittelt bekommt. Eine wichtige Rolle spielen dabei die ,unverfälschten Geräusche“, die mit dem O-Ton transportiert werden, denn „Das Gerede klingt spontan, Frage und Antwortspiele signalisieren Emotionalität; Diskussionen wirken lebhaft" bemerkt Haller (2013: 30) ${ }^{9}$. Somit ist das Interview im mündlichen Bereich verankert, was bei DaF-Lernenden zu einem leichteren, natürlicheren Sprach- und Kulturerwerb führt.

Ein weiterer Faktor, der hier verfolgt wurde, ist die Nähe zum Hörer, die zum einen durch das gewählte journalistische Genre als informeller/spontaner/nichtdidaktisierter Dialog, zum anderen durch den Gebrauch der sprachlichen Varietät zur Geltung kommt.

Beabsichtigt wird damit, die Studierenden mit einem ihnen bereits bekannten Alltagsphänomen erneut zu konfrontieren, ihnen neue Gesprächssituationen vorzuspielen, die auf Interagierende zurückgehen, die als Vermittler der hiesigen deutschen Kultur ${ }^{10}$ Wahrnehmungen, Denkweisen und Verhaltensmustern transponieren. Zudem soll das im Interview

8 Als ,frei formuliertes, spontanes Sprechen aus nicht gestellten, natürlichen Kommunikationssituationen“. Vgl. Schank, Gerd/Schoenthal, Gisela: Gesprochene Sprache. Eine Einführung in Forschungsansätze und Analysemethoden. Tübingen 1976, S. 7.

9 Haller, Michael: Das Interview. Konstanz 2013.

${ }^{10}$ Die deutsche Minderheit aus Rumänien setzt sich aus folgenden Bevölkerungsgruppen zusammen: Siebenbürger Sachen, Banater Schwaben, 
angesprochene Thema (Freiwilligenjahr) mit den Unterthemen (Paraguay, Mediasch/Mediaş, SOS-Kinderdorf, Studium in Deutschland) einen inter- und intrakulturellen Vergleich ermöglichen, der letztendlich zu einem besseren Verständnis der Andersartigkeit der anderen/fremden Kultur beiträgt und gleichzeitig neue Perspektiven eröffnet.

Das Radiointerview Ein Mediascher in Paraguay wurde als didaktisches Instrument in den Landeskundeunterricht (2 Seminarstunden im B-Fach) für Philologie-Studierende im 3. Semester, ohne Vorkenntnisse im Bereich Landeskunde und mit einem Sprachniveau von B1-B2, eingesetzt. Das Gespräch (9 Minuten) wurde von der Redakteurin Monica Strava für die Deutsche Sendung geführt. Interviewt wurde Raphael Toth aus Rumänien zum Thema Freiwilligendienst. Ort der Aufnahme: Mediasch/Mediaş, vermutlich draußen, in einem Kaffeehaus:

Freiwilligendienst, Au-Pair, Work and Travel - es gibt viele Möglichkeiten, nach dem Abitur erst einmal etwas völlig anderes zu machen und dabei neue Erfahrungen zu sammeln. Das veranlasste auch den Mediascher Raphael Toth, nach bestandener Abiturprüfung als Volontär in einem SOS-Kinderdorf in Paraguay zu arbeiten.

Er hat sich bewusst die Zeit genommen, um etwas Neues auszuprobieren und neue Seiten an sich zu entdecken. Er erlebte für acht Monate in Hohenau in Paraguay seine Stärken und Schwächen in ganz neuen Situationen, sammelte wichtige Erfahrungen und lernte viel dazu: Fähigkeiten und Fertigkeiten, die ihm im Studium sicherlich weiterhelfen werden. Was ihn dabei motivierte, nach Paraguay zu gehen und wie er den Aufenthalt vor Ort erlebt hat, das verriet er Monica Strava, die ihn in Mediasch traf. ${ }^{11}$

Sequenz 1: Das Interview wurde im Online-Unterricht ohne Vorentlastung vorgespielt, um die Studierenden zu motivieren und

Berglanddeutsche, Sathmarer Schwaben, Landler, Zipser, Buchenlanddeutsche, Dobrudschadeutsche und Altreichdeutsche.

${ }^{11}$ Vgl. https://www.radiobukarest.ro/2020/08/28/ein-mediascher-in-paraguay-2/\#more-1394; 10.05.2021. 
ihnen zu zeigen, was sie nach drei Semestern verstehen können, sodass diese über den Inhalt des Gesprächs im Voraus nicht informiert wurden.

Sequenz 2: Nach der ersten Auseinandersetzung mit dem Hörtext mussten die Studierenden das Thema des Dialogs erkennen und allgemeine Informationen (Geschlecht, Alter, sprachliche Herkunft, persönliche Interessen) über die interviewte Person liefern. Das Thema Freiwilligendienst/ Volontariat war für sie nicht neu, da man darüber im Rahmen des Sprachpraxisunterrichts diskutiert hatte.

Sequenz 3: Die Studierenden konnten das Interview ein zweites Mal hören und bestimmte Strukturen und Wörter aufschreiben, die ihnen aus dem Unterricht bekannt waren (z.B Volontariat, Freiwilligendienst/ -arbeit, soziales Engagement, Ausbildung, Studium usw). Dabei mussten sie auch angeben, welchen Sprecher ${ }^{12}$ sie besser verstanden und worin die Schwierigkeiten beim Verstehen bestanden haben.

Sequenz 4: Der in Sequenz 3 beschriebene Schritt wurde hier durch ein themenzentrietes Gespräch (deutsche Minderheit in Rumänien/Freiwilligenjahr) fortgesetzt. Die Studierenden setzten sich dann weiter mit dem Thema im Kurs kontrastiv auseinander.

Sequenz 5: Abschließend mussten die Kursteilnehmerinnen und -teilnehmer auf bestimmte Fragen eingehen: Sehen Sie vielleicht einen Zusammenhang zwischen der rumäniendeutschen Herkunft des Befragten und seine Entscheidung, für ein Jahr als Volontär in Paraguay im SOS-Kinderdorf mitzuwirken? Kann man sich gleich nach dem Abitur sozial engagieren, um erst später in das Studium einzusteigen? Gehört das Freiwilligenjahr zu einem bestimmten Kulturspezifikum? Würden Sie sich persönlich dafür die Zeit nehmen?

${ }^{12}$ Sowohl Interviewerin als auch Interviewter kommen aus Mediasch/ Mediaş. Beim Reden fallen jedoch bestimmte Besonderheiten auf: Bei der Interviewerin der rumäniendeutsche Hintergrund und beim Interviewten der bundesdeutsche Akzent. 


\section{Fazit}

Nach dem ersten Hören konnten die Studierenden das Gesprächsthema erkennen bzw. nennen und auch auf verschiedene Länder und Sprachenbezeichnungen eingehen. Eine einzige Kursteilnehmerin konnte Informationen über die genaue Herkunft der interviewten Person liefern. Die anderen hielten das Toponym Mediasch/Mediaş für ein sehr ,exotisches“ deutsches Wort. Die meisten Strukturen und Lexeme, die erkannt wurden, gehen auf das Lehrwerk von KlettDaF-Kompakt zurück. Die Studierenden lieferten Kommentare zur Aussprache der Interviewerin und meinten dabei, sie hätten Schwierigkeiten, ihre Fragen zu verstehen, da sie ,ein bisschen anders spreche" als andere deutschsprachigen Ansprechpartner, die sie bisher im Kurs gehört hatten. Nur ein paar Kursteilnehmerinnen konnten sich daran erinnern, etwas über die deutsche Minderheit in Rumänien im Rahmen einer Vorlesung gehört zu haben. Die meisten konnten sich nicht spontan zum Thema äußern, waren aber überrascht zu hören, dass es eine deutsche Minderheit in Rumänien gibt. Auch das Thema Freiwilligendienst nach dem Abitur war neu für sie, da es in Rumänien diesbezüglich keine Tradition gibt. Sie assoziierten das Thema mit etwas Fremdem oder betrachteten es als eine Besonderheit der „reichen“ Länder.

Die Beschäftigung mit einem journalistischen Interview im Landeskundeunterricht war für alle Studierenden neu, dafür aber ,sehr interessant" und „motivierend“. Viele bewerteten die gewählte Methode und die Arbeit mit dem mündlichen Dialog positiv, da dieser ,glaubwürdiger und interaktiver" als ein schriftlicher Dialog ist. Das Unterrichtsthema regte zu weiteren Diskussionen an.

Das journalistische Interview kann als didaktisches Instrument in den Landeskundeunterricht eingesetzt werden. Dazu kann die Lehrkraft Lückentexte und Übungen, die das Hörverstehen fördern oder weitere Übungen zur mündlichen Kommunikation, vorbereiten. Außerdem bieten solche Dialoge die 
Möglichkeit, unbekannte Kulturen zu entdecken und auch den (Sprach)Alltag der Menschen, die in Rumänien Deutsch als Muttersprache sprechen und außerhalb des geschlossenen Sprachraums in einer historisch gewachsenen Gemeinschaft leben, die ihre Traditionen und Werte seit Jahrhunderten pflegen. Der Status des Interviewten als Vertreter der deutschen Minderheit in Rumänien eröffnet DaF-Studierenden eine neue Sicht auf eine Sprachwelt, die real ist und uns aus der Nähe anspricht.

\section{Literaturverzechnis}

Altmayer, Claus: Landeskunde im Globalisierungskontext. Wozu noch Kultur im DaF-Unterricht? In: Haase, Peter/ Michaela Höller (Hgg.): Kulturelles Lernen im DaF-/DaZUnterricht. Paradigmenwechsel in der Landeskunde. Göttingen 2017.

Haller, Michael: Das Interview. Konstanz 2013.

Lăzărescu, Ioan: Rumäniendeutsch - eine eigenständige, jedoch bessere Varietät der deutschen Sprache. In: SchneiderWiejowski, Karina/Birte Kellermeier-Rehbein/Jakob Haselhuber (Hgg.): Vielfalt, Variation und Stellung der deutschen Sprache. Berlin 2013, S. 369-389.

Rösler, Dietmar: Deutsch als Fremdsprache. Eine Einführung. Stuttgart 2012.

Schank, Gerd/Schoenthal, Gisela: Gesprochene Sprache. Eine Einführung in Forschungsansätze und Analysemethoden. Tübingen 1976.

\section{Internetquellen}

Senghaas, Dieter: Kulturelle Globalisierung - ihre Kontexte, ihre Varianten. 2002; unterhttp://www.bpb.de/apuz/27019/ kulturelle-globalisierung-ihre-kontexte-ihrevarianten? $\mathrm{p}=$ all; 10.05 .2021 .

http://www.radiobukarest.ro; 10.05.2021.

https://www.radiobukarest.ro/2020/08/28/ein-mediascher-inparaguay-2/\#more-1394; 10.05.2021. 